\title{
CONSTRUCTIONS OF LAGRANGIAN COBORDISMS
}

\author{
SARAH BLACKWELL, NOÉMIE LEGOUT, CAITLIN LEVERSON, \\ MAŸLIS LIMOUZINEAU, ZIVA MYER, YU PAN, SAMANTHA PEZZIMENTI, \\ LARA SIMONE SUÁREZ, AND LISA TRAYNOR
}

\begin{abstract}
Lagrangian cobordisms between Legendrian knots arise in Symplectic Field Theory and impose an interesting and not well-understood relation on Legendrian knots. There are some known "elementary" building blocks for Lagrangian cobordisms that are smoothly the attachment of 0 - and 1-handles. An important question is whether every pair of nonempty Legendrians that are related by a connected Lagrangian cobordism can be related by a ribbon Lagrangian cobordism, in particular one that is "decomposable" into a composition of these elementary building blocks. We will describe these and other combinatorial building blocks as well as some geometric methods, involving the theory of satellites, to construct Lagrangian cobordisms. We will then survey some known results, derived through Heegaard Floer Homology and contact surgery, that may provide a pathway to proving the existence of nondecomposable (nonribbon) Lagrangian cobordisms.
\end{abstract}

\section{INTRODUCTION}

A contact manifold is an odd-dimensional manifold $Y^{2 n+1}$ together with a maximally non-integrable hyperplane distribution $\xi$. In a contact manifold, Legendrian submanifolds play a central role. These are the maximal integral submanifolds of $\xi: \Lambda^{n}$ such that $T_{p} \Lambda \subset \xi$, for all $p \in \Lambda$. In general, Legendrian submanifolds are plentiful and easy to construct. In this article we will restrict our attention to the contact manifold $\mathbb{R}^{3}$ with its standard contact structure $\xi=\operatorname{ker} \alpha$, where $\alpha=d z-y d x$. In this setting, every smooth knot or link has an infinite number of non-equivalent Legendrian representatives. More background on Legendrian knots is given in Section 2 .

The even-dimensional siblings of contact manifolds are symplectic manifolds. These are even-dimensional manifolds $M^{2 n}$ equipped with a closed, non-degenerate 2 -form $\omega$. In symplectic manifolds, Lagrangian submanifolds play a central role. Lagrangian submanifolds are the maximal dimensional submanifolds where $\omega$ vanishes on the tangent spaces: $L^{n}$ such that $\left.\omega\right|_{L}=0$. When the symplectic manifold is exact, $\omega=d \lambda$, it is important to understand the more restrictive subset of exact Lagrangians: these are submanifolds where $\left.\lambda\right|_{L}$ is an exact 1 -form. Geometrically, $L$ exact means that for any closed curve $\gamma \subset L, \int_{\gamma} \lambda=0$. In this article, we will restrict our attention to a symplectic manifold that is symplectomorphic to $\mathbb{R}^{4}$ with its standard symplectic structure $\omega_{0}=\sum d x_{i} \wedge d y_{i}$. In contrast to Legendrians, Lagrangians are scarce. For 
example, in $\mathbb{R}^{4}$ with its standard symplectic structure, the torus is the only closed surface that will admit a Lagrangian embedding into $\mathbb{R}^{4}$. A famous theorem of Gromov [Gro85] states that there are no closed, exact Lagrangian submanifolds of $\mathbb{R}^{4}$.

There has been a great deal of recent interest in a certain class of non-closed, exact Lagrangian submanifolds, known as Lagrangian cobordisms. These Lagrangian submanifolds live in the symplectization of a contact manifold and have cylindrical ends over Legendrians. In this article, we will focus on exact, orientable Lagrangian cobordisms from the Legendrian $\Lambda_{-}$to the Legendrian $\Lambda_{+}$that live in the symplectization of $\mathbb{R}^{3}$; this symplectization is $\mathbb{R} \times \mathbb{R}^{3}$ equipped with the exact symplectic form $\omega=d\left(e^{t} \alpha\right)$, where $t$ is the coordinate on $\mathbb{R}$ and $\alpha=d z-y d x$ is the standard contact form on $\mathbb{R}^{3}$. See Figure 5 for a schematic picture of a Lagrangian cobordism and Definition 1 for a formal definition. Such Lagrangian cobordisms were first introduced in Symplectic Field Theory (SFT) EGH00]: in relative SFT, we get a category whose objects are Legendrians and whose morphisms are Lagrangian cobordisms. Lagrangian fillings occur when $\Lambda_{-}=\emptyset$ and are key objects in the Fukaya category, which is an important invariant of symplectic four-manifolds. A Lagrangian cap occurs when $\Lambda_{+}=\emptyset$.

A basic question tied to understanding the general existence and behavior of Lagrangian submanifolds is to understand the existence of Lagrangian cobordisms: Given two Legendrians $\Lambda_{ \pm}$, when does there exist a Lagrangian cobordism from $\Lambda_{-}$to $\Lambda_{+}$? There are known to be a number of obstructions to this relation on Legendrian submanifolds coming from both classical and non-classical invariants of the Legendrians $\Lambda_{ \pm}$. Some of these obstructions are described in Section 2.3. To complement the obstructions, there are some known constructions. For example, it is well known [EG98, Cha10, EHK16] that there exists a Lagrangian cobordism between Legendrians $\Lambda_{ \pm}$that differ by Legendrian isotopy. In addition, by [EHK16, Cha12], it is known that there exists a Lagrangian cobordism from $\Lambda_{-}$to $\Lambda_{+}$if $\Lambda_{-}$can be obtained from $\Lambda_{+}$by a "pinch" move or if $\Lambda_{+}=\Lambda_{-} \cup U$, where $U$ denotes a Legendrian unknot with maximal Thurston-Bennequin number of -1 that is contained in the complement of a ball containing $\Lambda_{-}$. Topologically, between these slices, the cobordism changes by a saddle move (1-handle) and the addition of a local minimum (0-handle); see Figure 1. It is important to notice that there is not an elementary move corresponding to a local maximum (2-handle) move. By stacking these individual cobordisms obtained from isotopy, saddles, and minimums, one obtains what is commonly referred to as a decomposable Lagrangian cobordism. Through these moves, it is easy to construct Lagrangian cobordisms and fillings; see an example in Figure 7.

Towards understanding the existence of Lagrangians, it is natural to ask: Does there exist a Lagrangian cobordism from $\Lambda_{-}$to $\Lambda_{+}$if and only if there exists a decomposable Lagrangian cobordism from $\Lambda_{-}$to $\Lambda_{+}$? We know the answer to this question is "No": by studying the "movies" of the not necessarily 


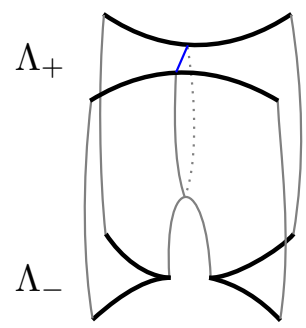

(A)

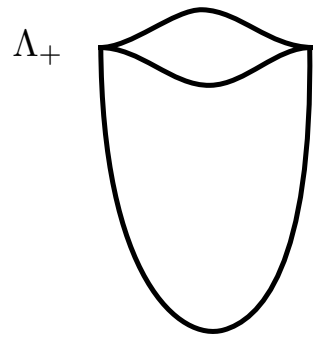

(B)

Figure 1. (A) The pinch move on $\Lambda_{+}$produces a Lagrangian saddle. (B) $\Lambda_{+}$obtained by introducing an unknotted component to $\Lambda_{-}$corresponds to the Lagrangian cobordism having a local min.

Legendrian slices of a Lagrangian. Sauvaget, Murphy, and Lin [Sau04, Lin16] have shown that there exists a genus two Lagrangian cap of the Legendrian unknot with Thurston-Bennequin number equal to -3 and rotation number 0 . The Lagrangian diagram moves used by [Lin16] to construct a Lagrangian cap are described in Section 3.3. The necessity of a local maximum when $\Lambda_{+} \neq \emptyset$ is not currently understood.

To formulate some precise motivating questions, we will use ribbon cobordism to denote a $2 n$-dimensional manifold that can be built from $k$-handles with $k \leq n$. This idea of restricting the handle index is well known in symplectic topology: Eliashberg [CE12, Oan15] has shown that any $2 n$-dimensional Stein manifold admits a handle decomposition with handles of dimension at most $n$, and thus any $2 n$-dimensional Stein cobordism between closed, $(2 n-1)$ dimensional contact manifolds must be ribbon. Working in the relative setting with submanifolds and using the handle decomposition from the "height" function given by the $\mathbb{R}$ coordinate on $\mathbb{R} \times \mathbb{R}^{3}$, we see that all decomposable 2-dimensional Lagrangian cobordisms between 1-dimensional Legendrian submanifolds are ribbon cobordisms. We are led to the following natural questions.

Motivating Questions. Suppose $\Lambda_{+} \neq \emptyset$ and there exists a connected Lagrangian cobordism $L$ from $\Lambda_{-}$to $\Lambda_{+}$. Then:

(1) Does there exist a decomposable Lagrangian cobordism from $\Lambda_{-}$to $\Lambda_{+}$?

(2) Does there exist a ribbon Lagrangian cobordism from $\Lambda_{-}$to $\Lambda_{+}$?

(3) Is L Lagrangian isotopic to a ribbon and/or decomposable Lagrangian cobordism?

There are some results known about Motivating Question (3) for the special case of the simplest Legendrian unknot. If $U$ denotes the Legendrian unknot with Thurston-Bennequin number -1, it is known that every (exact) Lagrangian filling is orientable [Rit09], and there is a unique (exact, orientable) Lagrangian filling of $U$ up to compactly supported Hamiltonian 
isotopy [EP96]. Moreover, any Lagrangian cobordism from $U$ to $U$ is Lagrangian isotopic, via a compactly supported Hamiltonian isotopy, to one in a countable collection given by the trace of a Legendrian isotopy induced by a rotation CDRGG.

Motivating Questions (1) and (2) are closely related and have deep ties to important questions in topology. Observe that a "yes" answer to (1) implies a "yes" to (2): if the existence of a Lagrangian cobordism implies the existence of a decomposable Lagrangian cobordism, then we also know the existence of a ribbon cobordism. Also note that when $\Lambda_{+}$is topologically a slice knot and $\Lambda_{-}=\emptyset,(2)$ is a symplectic version of the topological Slice-Ribbon conjecture: is every Lagrangian slice disk a ribbon disk? Cornwell, $\mathrm{Ng}$, and Sivek conjecture that the answer to Motivating Question (1) and (3) is "No": using the theory of satellites, we know that there is a Lagrangian concordance between $\Lambda_{ \pm}$shown in Figure 2, and in CNS16, Conjecture 3.3] it is conjectured that the concordance between the pair is not decomposable.
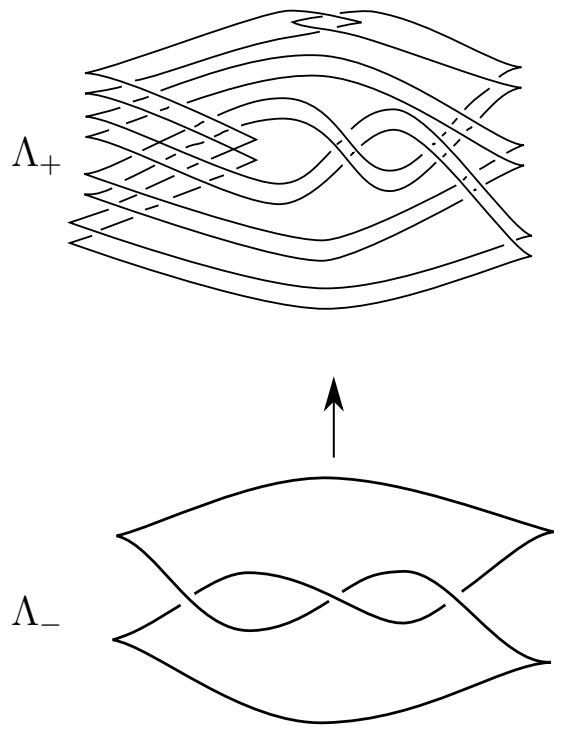

Figure 2. There is a Lagrangian concordance between these Legendrian knots that is conjectured to be non-decomposable. Here $\Lambda_{-}$is a Legendrian trefoil and $\Lambda_{+}$is a Legendrian Whitehead double of $m\left(9_{46}\right)$.

Very recently, Roberta Guadagni has discovered additional combinatorial moves that can be used to construct a "movie," meaning a sequence of slice pictures, of a Lagrangian cobordism; Figure 9 illustrates one of these tangle moves. With one of Guadagni's moves, it is possible to construct a movie of a Lagrangian cobordism between the Legendrians pictured in Figure 2; see Figure 10. Guadagni's moves are "geometric": they are developed through proofs similar to those used in the satellite procedure, and thus the handle attachments involved in the cobordism are not obvious. In particular, at this 
point it is not known if Guadagni's tangle moves are independent from the decomposable moves.

This survey article is organized as follows. In Section 2, we provide some background on Legendrians and Lagrangians, formally define Lagrangian cobordisms, and summarize known obstructions to the existence of Lagrangian cobordisms. In Section 3, we describe three "combinatorial" ways to construct Lagrangian cobordisms, and in Section 4, we describe more abstract "geometric" ways to construct Lagrangian concordances and cobordisms through satellites. Then in Section 5, we describe some potential pathways - through the theory of rulings, Heegaard-Floer homology, and contact surgery - to potentially show the existence of Legendrians that are Lagrangian cobordant but are not related by a decomposable Lagrangian cobordism.

Acknowledgements: This project was initiated at the workshop Women in Symplectic and Contact Geometry and Topology (WiSCoN) that took place at ICERM in July 2019. The authors thank the NSF-HRD 1500481 - AWM ADVANCE grant for funding this workshop. Leverson was supported by NSF postdoctoral fellowship DMS-1703356. We thank Emmy Murphy for suggesting and encouraging us to work on this project. In addition, we thank John Etnyre, Roberta Guadagni, Tye Lidman, Lenny Ng, Josh Sabloff, and Bülent Tosun for useful conversations related to this project.

\section{BACKGROUND}

2.1. Legendrian Knots and Links. In this section, we give a very brief introduction to Legendrian submanifolds in $\mathbb{R}^{3}$ and their invariants. More details can be found, for example, in the survey paper [Etn05].

In $\mathbb{R}^{3}$, the standard contact structure $\xi$ is a 2-dimensional plane field given by the kernel of the 1 -form $\alpha=d z-y d x$. In $\left(\mathbb{R}^{3}, \xi=\operatorname{ker} \alpha\right)$, a Legendrian knot is a knot in $\mathbb{R}^{3}$ that is tangent to $\xi$ everywhere. A useful way to visualize a Legendrian knot is to project it from $\mathbb{R}^{3}$ to $\mathbb{R}^{2}$. There are two useful projections: the Lagrangian projection

$$
\begin{aligned}
\pi_{L}: \quad \mathbb{R}^{3} & \rightarrow \mathbb{R}^{2} \\
(x, y, z) & \mapsto(x, y),
\end{aligned}
$$

as well as the front projection

$$
\begin{aligned}
& \pi_{F}: \quad \mathbb{R}^{3} \quad \rightarrow \quad \mathbb{R}^{2} \\
& (x, y, z) \mapsto(x, z) .
\end{aligned}
$$

An example of a Legendrian trefoil is shown in Figure 3.

Legendrian submanifolds are equivalent if they can be connected by a 1parameter family of Legendrian submanifolds. In fact, for each topological knot type there are infinitely many different Legendrian knots. Indeed, we can stabilize a Legendrian knot (as shown in Figure 4) to get another Legendrian knot of the same topological knot type. We can see that these are not Legendrian equivalent using Legendrian invariants. 

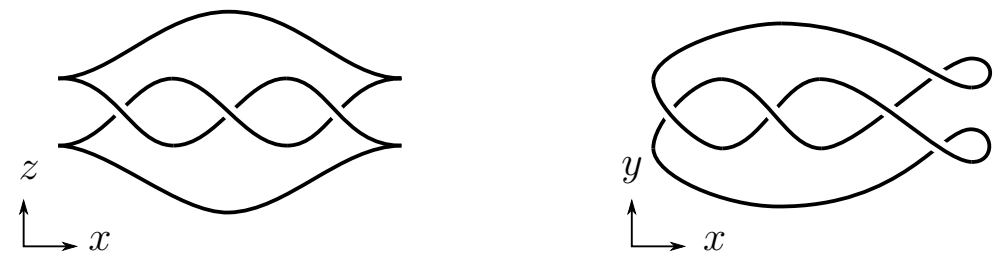

FiguRE 3. The front projection (left) and the Lagrangian projection (right) of a Legendrian trefoil.

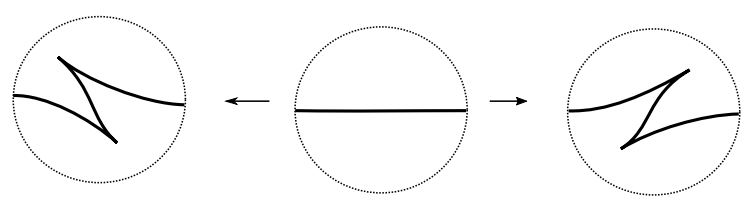

Figure 4. Two ways to stabilize a Legendrian knot in front projection.

Two useful classical invariants of Legendrian knots $\Lambda$ are the ThurstonBennequin number $t b(\Lambda)$ and the rotation number $r(\Lambda)$. They can be computed easily from front projections. Given the front projection of a Legendrian knot or link $\Lambda$, the Thurston-Bennequin number is

$$
t b(\Lambda)=\operatorname{writhe}\left(\pi_{F}(\Lambda)\right)-\#(\text { right cusps }),
$$

where the writhe is the number of crossings counted with sign. Once the Legendrian knot is equipped with an orientation, the rotation number is

$$
r(\Lambda)=\frac{1}{2}(\#(\text { down cusps })-\#(\text { up cusps })) \text {. }
$$

One can use these two invariants to see that stabilizations change the Legendrian knot type.

In future sections, we will not assume that our Legendrians $\Lambda_{ \pm}$come equipped with an orientation. In our Motivating Questions described in Section 1, our Lagrangian cobordisms are always orientable, so the existence of a Lagrangian cobordism from $\Lambda_{-}$to $\Lambda_{+}$will induce orientations on $\Lambda_{ \pm}$.

There are many powerful non-classical invariants that can be assigned to a Legendrian knot. Although this will not be a focus of this paper, we will give a brief description of some of these invariants. One important invariant stems from normal rulings, defined independently by Chekanov and Pushkar PC05] and Fuchs [Fuc03. A count of normal rulings leads to ruling polynomials PC05]; more details will be discussed in Section 5.1. Through the closely related theory of generating families, one can also associate invariant polynomials that record the dimensions of generating family homology groups [Tra01, JT06, FR11, ST13]. In addition, through the theory of pseudoholomoprhic curves, one can associate to a Legendrian $\Lambda$ a differential graded algebra (DGA), $\mathcal{A}(\Lambda)$ Che02, Eli98]. An augmentation is a DGA map from $\mathcal{A}(\Lambda)$ to a field. The count of augmentations is closely related to the count 
of ruling polynomials [Fuc03, NR13, NS06]. Augmentations can be used to construct finite-dimensional linearized contact homology groups [Che02, which are often known to be isomorphic to the generating family homology groups FR11. In addition, there are invariants for Legendrian knots coming from Heegaard Floer Homology [LOSS09] [OST08.

2.2. Lagrangian Cobordisms. Lagrangian cobordisms between Legendrian submanifolds always have "cylindrical ends" over the Legendrians, but other conditions vary: sometimes it is specified that the Lagrangian is exact, is embedded (or immersed), is orientable, or has a fixed Maslov class. In this paper, a Lagrangian cobordism is always exact, embedded, and orientable.

Definition 1. Let $\Lambda_{ \pm}$be two Legendrian knots or links in $\left(\mathbb{R}^{3}, \xi=\operatorname{ker} \alpha\right)$. A Lagrangian cobordism $L$ from $\Lambda_{-}$to $\Lambda_{+}$is an embedded, orientable Lagrangian surface in the symplectization $\left(\mathbb{R} \times \mathbb{R}^{3}, d\left(e^{t} \alpha\right)\right)$ such that for some $N>0$,

(1) $L \cap\left([-N, N] \times \mathbb{R}^{3}\right)$ is compact,

(2) $L \cap\left((N, \infty) \times \mathbb{R}^{3}\right)=(N, \infty) \times \Lambda_{+}$,

(3) $L \cap\left((-\infty,-N) \times \mathbb{R}^{3}\right)=(-\infty,-N) \times \Lambda_{-}$, and

(4) there exists a function $f: L \rightarrow \mathbb{R}$ and constant numbers $\mathfrak{c}_{ \pm}$such that $\left.e^{t} \alpha\right|_{T L}=d f$, where $\left.f\right|_{(-\infty,-N) \times \Lambda_{-}}=\mathfrak{c}_{-}$, and $\left.f\right|_{(N, \infty) \times \Lambda_{+}}=\mathfrak{c}_{+}$.

A Lagrangian filling of $\Lambda_{+}$is a Lagrangian cobordism with $\Lambda_{-}=\emptyset$; a Lagrangian cap of $\Lambda_{-}$is a Lagrangian cobordism with $\Lambda_{+}=\emptyset$. A Lagrangian concordance occurs when $\Lambda_{ \pm}$are knots and $L$ has genus 0 .

Figure 5 is a schematic representation of a Lagrangian cobordism.

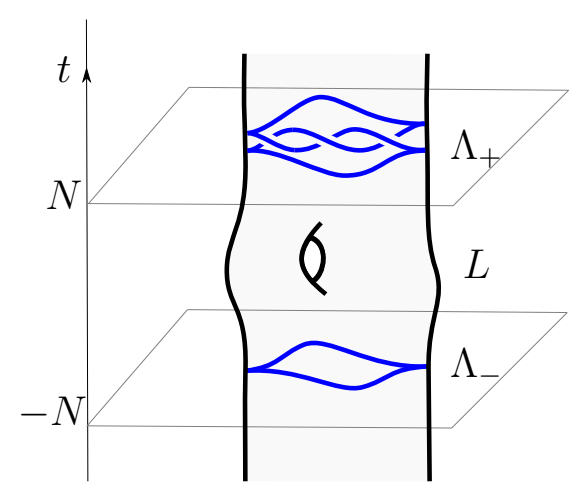

Figure 5. A Lagrangian cobordism from $\Lambda_{-}$to $\Lambda_{+}$.

Remark 1. In condition (4) of Definition 1, the fact that $\Lambda_{ \pm}$are Legendrian will guarantee that $f_{ \pm}$will be locally constant. Using this, it follows that any genus zero Lagrangian surface that is cylindrical over Legendrian knots will be exact. When $\Lambda_{ \pm}$have multiple components, one needs to check that the constant does not vary: this condition guarantees the exactness of the 
Lagrangian cobordism obtained by "gluing" together Lagrangian cobordisms Cha15a.

Remark 2. In contrast to topological cobordisms, Lagrangian cobordisms form a non-symmetric relationship on Legendrian knots Cha15b. In this article we will always denote the direction of increasing $\mathbb{R}_{t}$ coordinate by an arrow.

2.3. Obstructions to Lagrangian Cobordisms. The focus of this paper is on constructing Lagrangian cobordisms between two given Legendrians $\Lambda_{ \pm}$. In the smooth world, any two knots are related by a smooth cobordism, but in this more restrictive Lagrangian world, there are a number of obstructions that are important to keep in mind when trying to explicitly construct Lagrangian cobordisms. Here we mention a few that come from classical and non-classical invariants of the Legendrians $\Lambda_{ \pm}$.

\section{Obstructions:}

(1) If there exists a Lagrangian cobordism of genus $g$ between $\Lambda_{-}$and $\Lambda_{+}$, then there must exist a smooth cobordism of genus $g$ between the smooth knot types of $\Lambda_{-}$and $\Lambda_{+}$. Thus any obstruction of a smooth genus $g$ cobordism between $\Lambda_{-}$and $\Lambda_{+}$would obstruct a Lagrangian genus $g$ cobordism.

(2) Since there are no closed, exact Lagrangian surfaces [Gro85, if there exists a Lagrangian cap (respectively, filling) for $\Lambda$, then there cannot exist a Lagrangian filling (respectively, cap) of $\Lambda$.

(3) As shown in Cha10, if there exists a Lagrangian cobordism $L$ from $\Lambda_{-}$to $\Lambda_{+}$, then

$$
r\left(\Lambda_{-}\right)=r\left(\Lambda_{+}\right) \quad \text { and } \quad t b\left(\Lambda_{+}\right)-t b\left(\Lambda_{-}\right)=-\chi(L) .
$$

In particular, if a Legendrian knot $\Lambda$ admits a Lagrangian filling or cap, then $r(\Lambda)=0$. Also, combining this equality on $t b$ and the slice-Bennequin inequality [Rud97], we see that, when $\Lambda$ is a single component knot, if there exists a Lagrangian cap $L$ of $\Lambda$, then $t b(\Lambda) \leq$ -1 and $g(L) \geq 1$.

(4) If there exists a Maslov 0 ([EES05]) Lagrangian cobordism $\Sigma$ from $\Lambda_{-}$ to $\Lambda_{+}$, and $\Lambda_{-}$has an augmentation, then

(a) $\# \operatorname{Aug}\left(\Lambda_{+} ; \mathbb{F}_{2}\right) \geq \# A u g\left(\Lambda_{-} ; \mathbb{F}_{2}\right)$, where $\mathbb{F}_{2}$ is the finite field of two elements, and $\# \operatorname{Aug}\left(\Lambda ; \mathbb{F}_{2}\right)$ denotes the number of augmentations of $\Lambda$ to $\mathbb{F}_{2}$ up to DGA homotopy [Pan17, $\left.\mathrm{CSLL}^{+} 20\right]$, and

(b) the ruling polynomials $R_{\Lambda_{ \pm}}(z)$ (see Section 5.1 for definitions) satisfy

$$
R_{\Lambda_{-}}\left(q^{1 / 2}-q^{-1 / 2}\right) \leq q^{-\chi(\Sigma) / 2} R_{\Lambda_{+}}\left(q^{1 / 2}-q^{-1 / 2}\right),
$$

for any $q$ that is a power of a prime number [Pan17].

(5) If $\Lambda$ admits a Maslov 0 Lagrangian filling $L$, and if $\epsilon_{L}$ denotes the augmentation of $\Lambda$ induced by $L$, then $L C H_{\epsilon_{L}}^{k}(\Lambda) \cong H_{n-k}(L)$, which is known as the Ekholm-Seidel isomorphism [Ekh12, and whose proof was completed by Dimitroglou Rizell in DR16. More generally, if 
there is a cobordism from $\Lambda_{-}$to $\Lambda_{+}$, and if $\Lambda_{-}$admits an augmentation, then CDRGG20 provides several long exact sequences relating the homology of the cobordism and the Legendrian contact (co)homologies of its Legendrian ends. A version of this isomorphism and these long exact sequences using generating families are given in [ST13.

(6) If $\Lambda$ admits an augmentation, $\Lambda$ does not admit a Lagrangian cap, as the augmentation implies the non-acyclicity of the DGA $\mathcal{A}(\Lambda)$ EES09, Theorem 5.5], and from [DR15, Corollary 1.9] if a Legendrian admits a Lagrangian cap then its DGA $\mathcal{A}(\Lambda)$ (with $\mathbb{Z}_{2}$ coefficients) is acyclic.

There are additional obstructions, obtained through Heegaard Floer Theory, that can be used to obstruct Lagrangian concordances and cobordisms BSar, GJ19, BLWar. Some of these will be discussed more in Section 5.3.

Remark 3. Observe that the obstructions in (4) and (6) assume that the bottom $\Lambda_{-}$has an augmentation, and stabilized knots will never have an augmentation. It would be nice to have more obstructions when $\Lambda_{-}$is a stabilized knot. This might be possible using the theory of "satellites" described in Section 4.1: it is possible for the satellite of a stabilized Legendrian to admit an augmentation. See Section 4.3 for more discussions in this direction.

\section{Combinatorial Constructions of Lagrangian Cobordisms}

A convenient way of visualizing topological cobordisms is through "movies": a sequence of pictures that represent slices of the Lagrangian. In this section, we describe three known combinatorial ways to construct Lagrangian cobordisms through such an approach.

3.1. Decomposable Moves. It is well known that if $\Lambda_{-}$and $\Lambda_{+}$are Legendrian isotopic, then there exists a Lagrangian cobordism from $\Lambda_{-}$to $\Lambda_{+}$; see, for example, [EG98, Cha10, EHK16]. Isotopy, together with two types of handle moves, form the basis for decomposable Lagrangian cobordisms.

Theorem 2 ([EHK16, BST15]). If the front diagrams of two Legendrian links $\Lambda_{-}$and $\Lambda_{+}$are related by any of the following moves, there is a Lagrangian cobordism $L$ from $\Lambda_{-}$to $\Lambda_{+}$.

Isotopy: There is a Legendrian isotopy between $\Lambda_{-}$and $\Lambda_{+}$; see Figures $6($ a) $-6($ c) for Reidemeister Move I-III.

1-handle: The front diagram of $\Lambda_{-}$can be obtained from the front diagram of $\Lambda_{+}$by "pinching" two oppositely-oriented strands; see Figure $6(d)$. We will also refer to this move as a "Pinch Move."

0-handle: The front diagram of $\Lambda_{-}$can be obtained from the front diagram of $\Lambda_{+}$by deleting a component of $\Lambda_{+}$that is the front diagram of a standard Legendrian unknot $U$ with maximal Thurston-Bennequin number of -1 as long as there exist disjoint disks $D_{U}, D_{U^{c}} \subset \mathbb{R}_{x z}^{2}$ containing the xz-projection of $U$ and the other components of $\Lambda_{+}$, respectively. Such an "unknot filling" can be seen in Figure 6(e). 


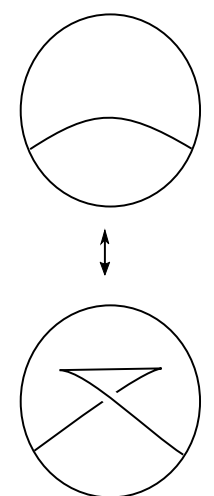

(a)

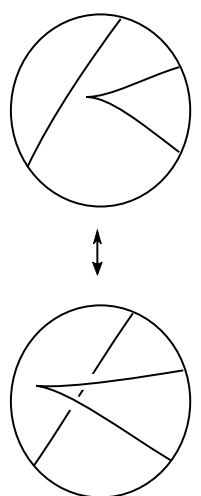

(b)

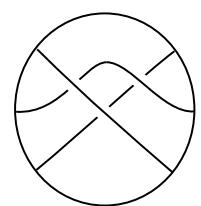

$\uparrow$

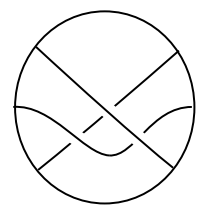

(c)
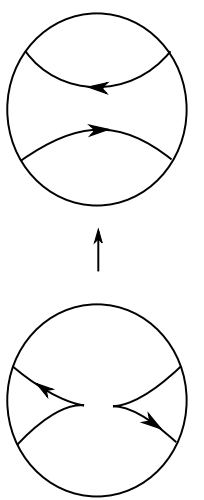

(d)

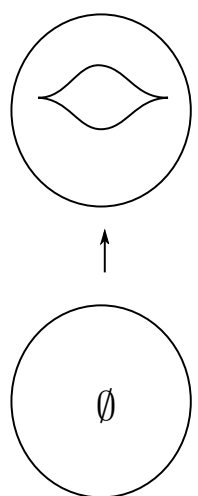

(e)

Figure 6. Decomposable moves in terms of front projections. Arrows indicate the direction of increasing $\mathbb{R}_{t}$ coordinate in the symplectization. The move in $(b)$ only shows the Reidemeister II move in the left cusp case, but there is an analogous move for the right cusp.

Definition 3. A Lagrangian cobordism $L$ from $\Lambda_{-}$to $\Lambda_{+}$is called elementary if it arises from isotopy, a single 0-handle, or a single 1-handle. A Lagrangian cobordism $L$ from $\Lambda_{-}$to $\Lambda_{+}$is decomposable if it is obtained by stacking elementary Lagrangian cobordisms.

Observe that there is not an elementary move corresponding to a 2-handle (maximum). Also note that the elementary 1-handle (saddle) move can be used to connect two components or to split one component into two.

Decomposable cobordisms are particularly convenient as they are easy to describe in a combinatorial fashion, through a list of embedded Legendrian curves,

$$
\Lambda_{-}=\Lambda_{0} \rightarrow \Lambda_{1} \rightarrow \cdots \rightarrow \Lambda_{n}=\Lambda_{+},
$$

where the front projection of the Legendrian $\Lambda_{i+1}$ is related to that of $\Lambda_{i}$ by isotopy or one of the 0-handle or 1-handle moves.

Example 4. One can construct a Lagrangian filling of a positive Legendrian trefoil with maximal Thurston-Bennequin number using the series of moves shown in Figure 7: a 0-handle, followed by three Reidemeister I moves, followed by two 1-handles (or pinch moves). This gives a genus 1 (orientable, exact) Lagrangian filling of this Legendrian trefoil. Since we are assuming that Lagrangian fillings and caps are always exact, this implies that this trefoil cannot admit a Lagrangian cap; see Section 2.3 Obstructions (2).

Example 5. Using elementary moves, one can also construct a Lagrangian concordance from the unknot with $t b=-1$ to a Legendrian representative of the knot $m\left(9_{46}\right)$, as shown on Figure 8 .

3.2. Guadagni Moves. Very recently, Roberta Guadagni has discovered a new "tangle" move; see Figure 9. This is not a local move: there are some 


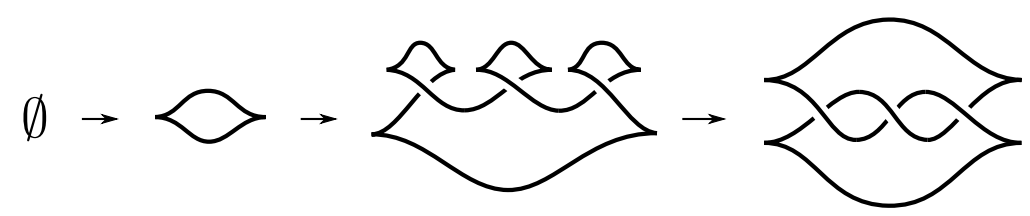

FiguRE 7. A decomposable Lagrangian filling of a Legendrian trefoil.

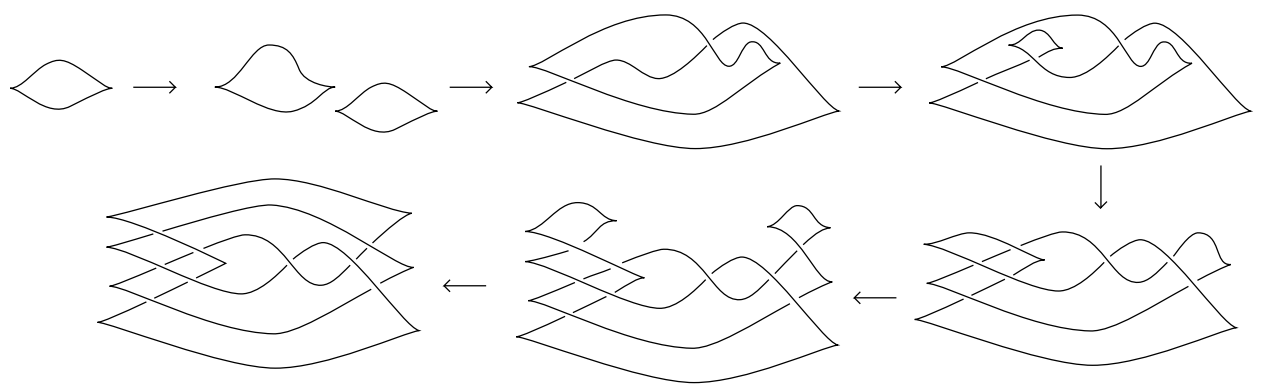

Figure 8. A decomposable Lagrangian cobordism from a Legendrian unknot to a Legendrian $m\left(9_{46}\right)$.

global requirements. In particular, this move cannot be applied if all components of the tangle are contained in the same component of $\Lambda_{-}$: the component of $\Lambda_{-}$containing the blue strand must be different than the components containing the other strands of the tangle.

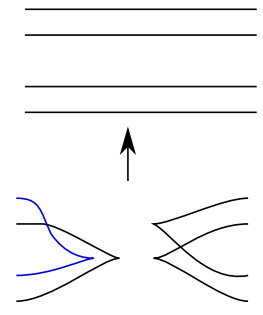

FiguRE 9. Under some global conditions, there exists a Lagrangian cobordism between these tangles.

Example 6. With Guadagni's tangle move, it is possible to construct a Lagrangian cobordism between the Legendrians pictured in Figure 2; see Figure 10. However, at this point it is not known if Guadagni's tangle move is independent of the decomposable moves.

3.3. Lagrangian Diagram Moves. As shown in Section 3.1, decomposable cobordisms are constructed from 0-handles and some 1-handles (saddles) but no 2-handles (caps). Based on the work of Sauvaget [Sau04], Lin [Lin16] constructs a genus two cap of a twice stabilized unknot, and thus gives the first explicit example of a non-decomposable Lagrangian cobordism. The construction describes time-slices of a Lagrangian cobordism through a list of moves on "decorated Lagrangian diagrams." 


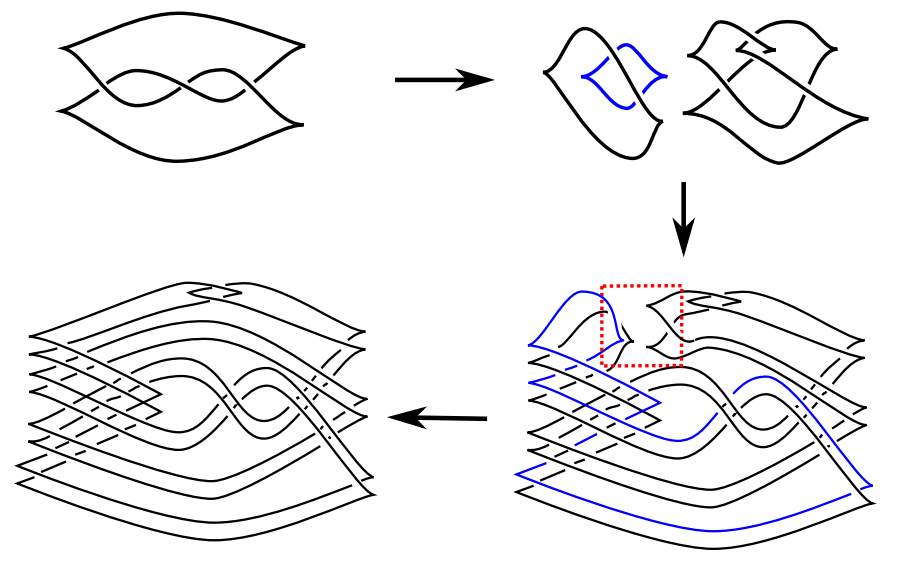

Figure 10. A movie, using a Guadagni move, of an (orientable, exact) Lagrangian cobordism from the trefoil to the Whitehead double of $m\left(9_{46}\right)$ in Figure 2 .

A decorated Lagrangian diagram is a curve in the $x y$-plane with the compact regions decorated by a positive number, which is the area of the region. Figure 11 shows some examples: in the illustration of the $F$ move, $U$ is a Lagrangian projection of the Legendrian unknot with maximal ThurstonBennequin number; in the illustration of the $C$ move, $U_{m}$ is a decorated Lagrangian diagram, but is not the Lagrangian projection of a Legendrian knot.

Theorem 7 ([Lin16]). Let $\Lambda_{ \pm}$be Legendrian links and $D_{ \pm}$be their corresponding decorated Lagrangian projections. If one can create a sequence of decorated Lagrangian diagrams

$$
D_{-}=D_{0} \rightarrow D_{1} \rightarrow \cdots \rightarrow D_{n}=D_{+}
$$

such that each diagram $D_{i+1}$ can be obtained from $D_{i}$ by the following combinatorial moves, then there is a compact Lagrangian submanifold in $\mathbb{R} \times \mathbb{R}^{3}$ with boundary $\Lambda_{-} \cup \Lambda_{+}$, where $\Lambda_{ \pm} \subset\{ \pm N\} \times \mathbb{R}^{3}$, for some $N>0$.

(1) $R_{0}$ : a planar isotopy that changes areas by the amount $\pm A$, for $A>0$. This operation can only be done in the direction specified.

(2) $R_{2}$ : a Reidemeister II move. One can either introduce or eliminate two crossings assuming some area conditions are satisfied: it is possible to introduce or remove two crossings as long as the area of the inner region, denoted by 0 in the diagram, is less than either the area $\delta$ or the area $\eta$. One can also do this move with the lower strand passing under the upper strand.

(3) $R_{3}$ : a Reidemeister III move. One can perform a Reidemeister III move as long as the area of the inner region, denoted by 0 in the diagram, is less than either the area $\epsilon$, the area $\delta$ or the area $\eta$. The fixed center crossing can be reversed. Additionally, the moving strand can also occur as an overstrand. 

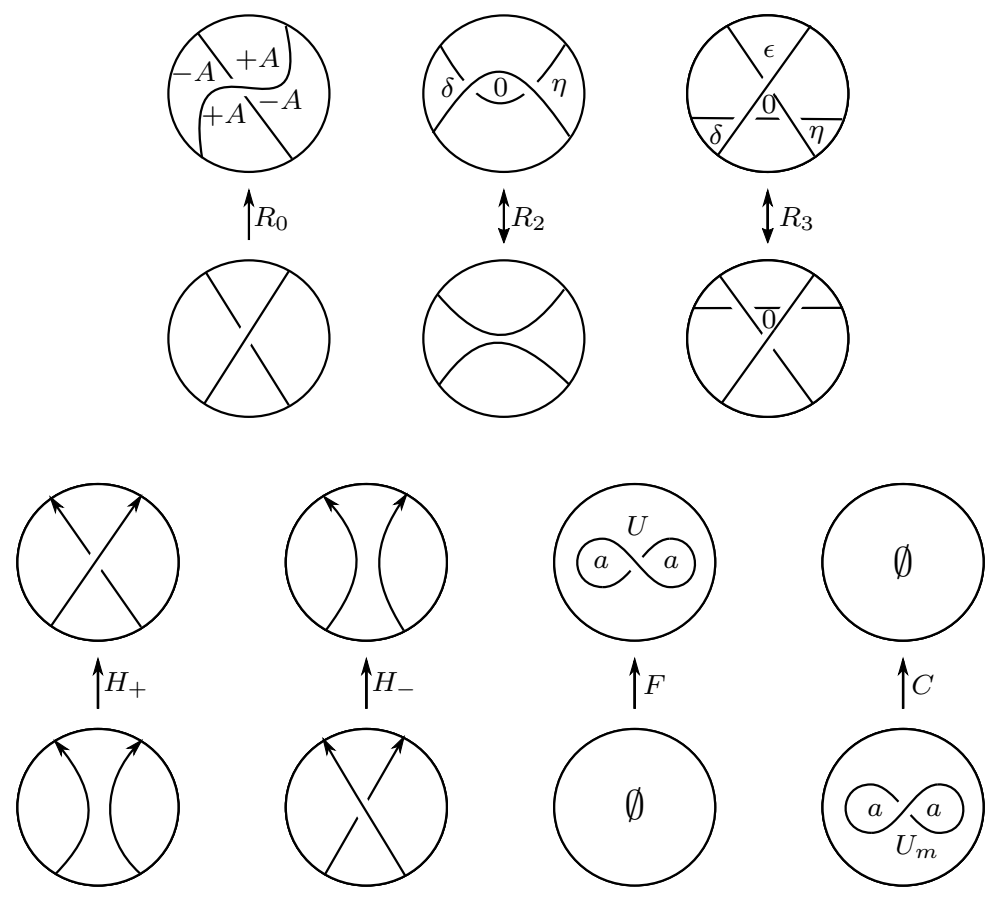

Figure 11. The Lagrangian diagram moves. The labels in $R_{0}$ move represent the change of area through the move, while other labels $0, \epsilon, \delta, \eta, a$ indicate the area of the corresponding regions; here 0 represents a positive area that is smaller than either the area $\epsilon$, the area $\delta$ or the area $\eta$.

(4) $H_{+}$: a handle attachment that creates a positive crossing in the diagram.

(5) $H_{-}:$a handle attachment that removes a negative crossing in the diagram.

(6) F: a filling that creates the diagram U, which is the Lagrangian projection of an unknot with maximal Thurston-Bennequin number.

(7) $C$ : a cap that eliminates the diagram $U_{m}$, which is the topological mirror of $U$.

These moves are called Lagrangian diagram moves. Moreover, the constructed Lagrangian will be exact if, in addition,

(E1) Each move results in a diagram with all components having a total signed area equal to 0 . The signed area of a region is determined by the sum of the signed heights of its Reeb chords.

(E2) If a handle attachment merges two components of a link, the components being merged must be vertically split, meaning that the images of the xy-projections of these components are contained in disjoint disks. 
Remark 4. (1) For condition (E2), the $H_{-}$can never be applied to merge components, and $H_{+}$can only be applied if the components being merged are vertically split.

(2) A main distinction between the Lagrangian diagram moves and the decomposable moves is that each diagram $D_{i}$ in the middle of the sequence is not necessarily the Lagrangian projection of a Legendrian link. They are just the $x y$-projection of some time $t_{i}$-slice of the cobordism. Thus the Lagrangian diagram moves are more flexible than the decomposable moves. However, keeping track of the areas is an added complication.

Example 8. Figure 12 illustrates the construction of a Lagrangian torus using the Lagrangian diagram moves. This torus fails to be exact since condition (E1) is violated. Figure 13 gives another construction of a Lagrangian torus. This time, all components have signed area 0, but now condition (E2) is violated.

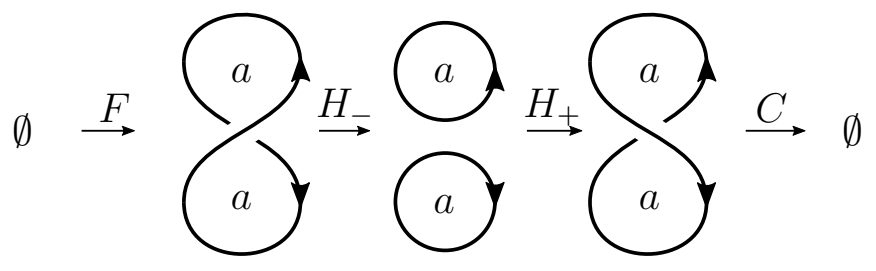

FiguRE 12. A (non-exact) Lagrangian torus constructed using the Lagrangian diagram moves. The middle figure violates (E1).

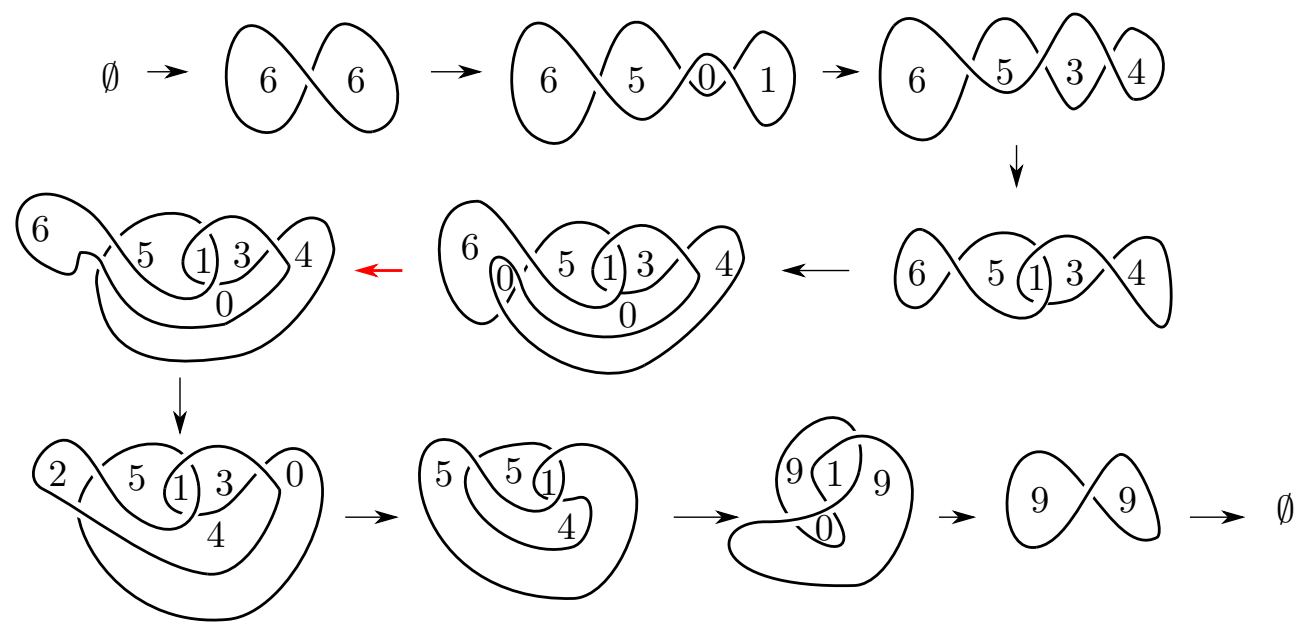

FIGURE 13. A (non-exact) Lagrangian torus constructed using the Lagrangian diagram moves. These figures satisfy (E1) but (E2) is violated in the step labelled by a red arrow. 


\section{Geometrical Constructions of Lagrangian Cobordisms}

An important general way to know of the existence of Lagrangian cobordisms without using the constructions described in Section 3 comes through the satellite operation. In this section, we review the satellite construction and then state results from [CNS16, GSY20] about the existence of a Lagrangian concordance/cobordism from $\Lambda_{-}$to $\Lambda_{+}$implying the existence of a Lagrangian concordance/cobordism between corresponding satellites.

4.1. The Legendrian Satellite Construction. We begin by reviewing the construction of a Legendrian satellite; see also [NT04, Appendix] and [CNS16, Section 2.2]. To construct a Legendrian satellite, begin by identifying the open solid torus $S^{1} \times \mathbb{R}^{2}$ with the 1 -jet space of the circle, $J^{1} S^{1} \cong T^{*} S^{1} \times \mathbb{R}$, equipped with the contact form $\alpha=d z-y d x$, where $x, y$ are the coordinates in $T^{*} S^{1}$ and $z$ is the coordinate in $\mathbb{R}$. Similar to the situation for $\mathbb{R}^{3} \cong J^{1} \mathbb{R}$, we can recover a Legendrian knot in $J^{1} S^{1}$ from its front projection in $S_{x}^{1} \times \mathbb{R}_{z}$, which is typically drawn by representing $S^{1}$ as an interval with its endpoints identified.

Given an oriented Legendrian companion knot $\Lambda \subset \mathbb{R}^{3}$ and a oriented Legendrian pattern knot $P \subset J^{1}\left(S^{1}\right)$, the Legendrian neighborhood theorem says that $\Lambda$ has a standard neighborhood $N(\Lambda)$ such that there is a contactomorphism $\kappa: J^{1}\left(S^{1}\right) \rightarrow N(\Lambda)$. The Legendrian satellite, $S(\Lambda, P)$, is then the image $\kappa(P)$. The front projection of $S(\Lambda, P)$ is as shown in Figure 14. In particular, suppose that the front projection of the pattern $P$ intersects the vertical line at the boundary of the $S^{1}$ interval $n$ times. We then make an $n$-copy of $\Lambda$ by using $n$-disjoint copies of $\Lambda$ that all differ by small translations in the $z$-direction. Take a point on the front projection of $\Lambda$ that is oriented from left to right, cut the front of the $n$-copy open along the $n$-copy at that point, and insert the front diagram of $P$. The orientation on the satellite $S(\Lambda, P)$ is induced by the orientation on $P$.

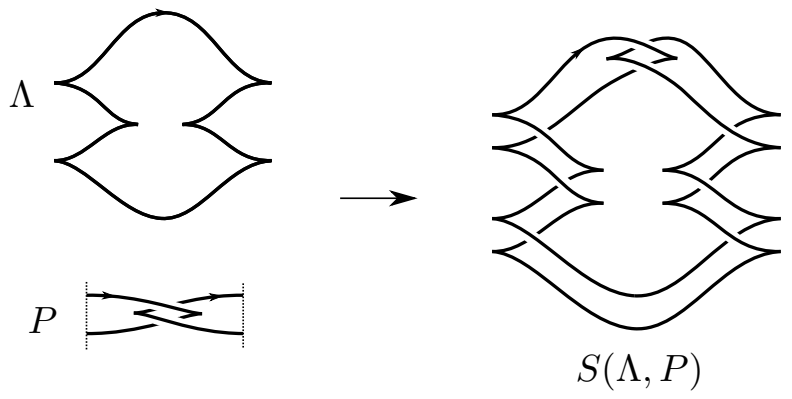

Figure 14. A example of Legendrian satellite.

Remark 5. The satellite operation often makes Legendrian knots "nicer"; for example, in Figure 14, the companion $\Lambda$ is stabilized and does not admit an augmentation or a normal ruling. However, the satellite $S(\Lambda, P)$ does admit a normal ruling and augmentation. 
4.2. Lagrangian Cobordisms for Satellites. In CNS16, Theorem 2.4], Cornwell, Ng, and Sivek, show that Lagrangian concordance is preserved by the Legendrian satellite operation.

Theorem 9 ([CNS16]). Suppose $P \subset J^{1} S^{1}$ is a Legendrian knot. If there exists a Lagrangian concordance $L$ from a Lengendrian knot $\Lambda_{-}$to a Lengendrian knot $\Lambda_{+}$, then there exists a Lagrangian concordance $L_{P}$ from $S\left(\Lambda_{-}, P\right)$ to $S\left(\Lambda_{+}, P\right)$.

In particular, as shown in Figure 8, there is a Lagrangian concordance from $\Lambda_{-}$, which is the Legendrian unknot with $t b=-1$, to $\Lambda_{+}$, which is the Legendrian $m\left(9_{46}\right)$ with maximal $t b=-1$. Using the Legendrian "clasp" tangle $P$ as shown in Figure 14 - which produces the Legendrian Whitehead double - we can conclude that there exists a Lagrangian concordance from $S\left(\Lambda_{-}, P\right)$ to $S\left(\Lambda_{+}, P\right)$. In fact, $S\left(\Lambda_{-}, P\right)$ is the positive trefoil with $t b=1$. Thus Theorem 9 implies that there exists a Lagrangian concordance between the Legendrian knots in Figure 2.

Conjecture 10 (CNS16, Conjecture 3.3]). The Lagrangian concordance from $S\left(\Lambda_{-}, P\right)$ to $S\left(\Lambda_{+}, P\right)$ built through the satellite construction is not decomposable.

Theorem 9 has been extended to higher genus cobordisms by Guadagni, Sabloff, and Yacavone in GSY20]. To state their theorem, we need to first introduce the notion of "twisting" and then closing a tangle $T \subset J^{1}[0,1]$. Given a Legendrian tangle $T \subset J^{1}[0,1], \Delta T$ is the tangle obtained by adding the tangle $T$ and the full twist tangle $\Delta$, which is illustrated in Figure 15 the tangle $\Delta^{t} T$ can be thought of as $T$ followed by $t$ full twists. Given a Legendrian tangle $T \subset J^{1}[0,1], \bar{T} \subset J^{1}\left(S^{1}\right)$ will denote the associated closure to a Legendrian link.

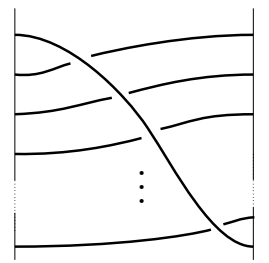

Figure 15. For an $n$-stranded tangle, repeating this basic tangle $n$ times produces a full twist.

Theorem 11 (GSY20]). Suppose $T \subset J^{1}[0,1]$ is a Legendrian tangle whose closure $\bar{T} \subset J^{1}\left(S^{1}\right)$ is a Legendrian knot. If there exists a Lagrangian cobordism $L$ from $\Lambda_{-}$to $\Lambda_{+}$of genus $g(L)$, then there exists a Lagrangian cobordism $L_{T}$ from $S\left(\Lambda_{-}, \overline{\Delta^{2 g(L)+1} T}\right)$ to $S\left(\Lambda_{+}, \overline{\Delta T}\right)$.

In fact, Theorem 11 can be generalized to use the closure of different tangles $T_{-}$and $T_{+}$that are Lagrangian cobordant; for details, see [GSY20]. 
Remark 6. It is natural to wonder if, along the lines of Conjecture 10, this higher genus satellite procedure can create additional candidates for Legendrians that can be connected by a Lagrangian cobordism but not by a decomposable Lagrangian cobordism. In [GSY20, Theorem 1.5], it is shown that if the cobordism $L$ from $\Lambda_{-}$to $\Lambda_{+}$is decomposable and the handles in the decomposition satisfy conditions known as "Property A", then the corresponding satellites $S\left(\Lambda_{-}, \Delta^{2 g(L)+1} P\right)$ and $S\left(\Lambda_{+}, \Delta P\right)$ will also be connected by a decomposable Lagrangian cobordism. In particular, if there exists a decomposable cobordism $L$ that does not satisfy Property A and is not isotopic to a cobordism that satisfies Property A, then the satellite construction would lead to a higher genus candidate that generalizes Conjecture 10 .

4.3. Obstructions to Cobordisms through Satellites. In Section 2.3, some known obstructions to the existence of a Lagrangian cobordism were mentioned. As mentioned in Remark 3, a number of these obstructions require $\Lambda_{-}$to admit an augmentation, and thus in particular $\Lambda_{-}$must be nonstabilized. However, as mentioned in Remark 5, it is possible for the satellite of a Legendrian $\Lambda$ to admit an augmentation even if $\Lambda$ does not. So the contrapositive of Theorem 9 provides a potential strategy for further obstructions to the existence of a Lagrangian cobordism when $\Lambda_{-}$does not admit an augmentation. For example, motivated by Obstruction (4) in Section 2.3. one can ask: Can a count of augmentations give an obstruction to the existence of a Lagrangian concordance from $S\left(\Lambda_{-}, P\right)$ to $S\left(\Lambda_{+}, P\right)$ and thereby obstruct the existence of a Lagrangian concordance from $\Lambda_{-}$to $\Lambda_{+}$? In fact, this augmentation count will not likely provide a further obstruction: a simple computation shows that when $\Lambda$ is stabilized enough, the number of augmentations of $S(\Lambda, P)$ only depends on the Legendrian pattern $P$. If trying to pursue this path to obtain further obstructions to Lagrangian cobordisms, it is useful to keep in mind the following result of $\mathrm{Ng}$ that shows the DGA of the satellite of a Legendrian $\Lambda$ might only remember the underlying knot type of $\Lambda$.

Theorem 12 (|Ng01]). Suppose $\Lambda_{1}$ and $\Lambda_{2}$ are stabilized Legendrian knots that are of the same topological knot type and have the same Thurston-Bennequin and rotation numbers. For a Legendrian pattern $P$ whose front intersects a vertical line by two points, the DGAs of $S\left(\Lambda_{1}, P\right)$ and $S\left(\Lambda_{2}, P\right)$ are equivalent.

\section{Candidates for Non-Decomposable Lagrangian Cobordisms}

Now that we have developed some ways to construct a Lagrangian cobordism through combinatorial moves and satellites, we state some theorems that show if a Lagrangian cobordism does exist, then it cannot be decomposable: this addresses Motivating Question (1). While we discuss these theorems, it is useful to keep in mind the known obstructions to Lagrangian cobordisms that were mentioned in Section 2.3 . 


\subsection{Candidates for Non-decomposable Lagrangian Cobordisms from}

Normal Rulings. One simple way to show that two Legendrians $\Lambda_{ \pm}$cannot be connected by a decomposable Lagrangian cobordism comes from a count of "combinatorial" rulings. Roughly, a normal ruling of a Legendrian $\Lambda$ is a "decomposition" of the front projection into pairs of paths from left cusps to right cusps such that

(1) each pair of paths starts from a common left cusp and ends at a common right cusp, has no further intersections, and bounds a topological disk whose boundary is smooth everywhere other than at the cusps and certain crossings called switches, and

(2) near a switch, the pair of paths must be arranged as in one of the diagrams in Figure 16; observe that near the switch, vertical slices of the associated disks are either disjoint or the slices of one are contained in the slices of the other.

Formal definitions of normal rulings can be found in, for example, [PC05] and [Fuc03].
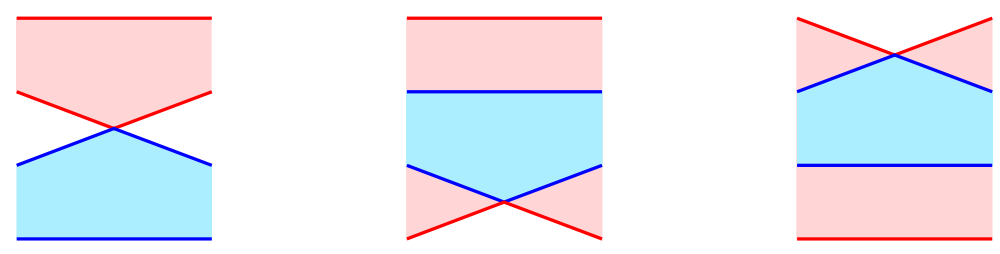

FiguRE 16. Normal rulings near a switch.

As an illustration, all normal rulings of a particular Legendrian trefoil are shown in Figure 17.
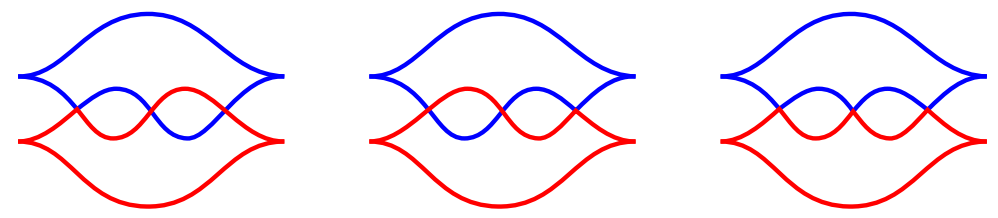

FIGURE 17. All normal rulings of this max $t b$ positive Legendrian trefoil.

For each normal ruling $R$, let $s(R)$ and $d(R)$ be the number of switches and number of disks, respectively. By [PC05], the ruling polynomial is

$$
R_{\Lambda}(z)=\sum_{R} z^{s(R)-d(R)},
$$

where the sum is over all the normal rulings, is an invariant of $\Lambda$ under Legendrian isotopy. Normal rulings and augmentations are closely related even though they are defined in very different ways [Fuc03, FI04, NS06, Sab05]. 
We have the following obstruction to decomposable cobordisms in terms of normal rulings.

Theorem 13. If $\Lambda_{-}$has $m$ normal rulings and $\Lambda_{+}$has $n$ normal rulings with $m>n$, then there is no decomposable Lagrangian cobordism from $\Lambda_{-}$to $\Lambda_{+}$.

Proof. One can compare the number of normal rulings of the two ends for the decomposable moves, as shown in Figure 18. Thus any normal ruling of $\Lambda_{-}$ induces a normal ruling of $\Lambda_{+}$. Different normal rulings of $\Lambda_{-}$induce different normal rulings of $\Lambda_{+}$. Therefore the number of normal rulings of $\Lambda_{+}$is bigger than or equal to the number of normal rulings of $\Lambda_{-}$.
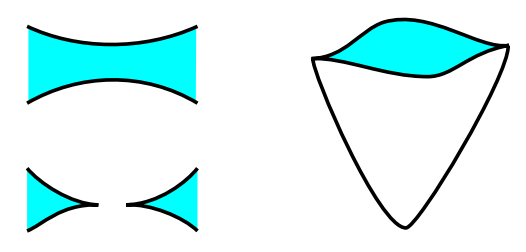

Figure 18. Comparison of normal rulings for decomposable moves.

Here is a strategy to show the existence of Legendrians that can be connected by a Lagrangian cobordism but not by one that is decomposable.

Strategy 1. Choose Legendrians $\Lambda_{ \pm}$such that:

(1) $\Lambda_{+}$has fewer graded normal rulings than $\Lambda_{-}$, and

(2) it is possible to construct, via a combination of the combinatorial constructions from Section 3 or the satellite construction from Section 4. a Lagrangian cobordism from $\Lambda_{-}$to $\Lambda_{+}$.

Remark 7 . If $\Lambda_{ \pm}$admit normal rulings, they will admit augmentations [FI04, Sab05. From Section 2.3 obstructions (4)b, we then know that if there is a Lagrangian cobordism from $\Lambda_{-}$to $\Lambda_{+}$, their ruling polynomials satisfy

$$
R_{\Lambda_{-}}\left(q^{1 / 2}-q^{-1 / 2}\right) \leq q^{-\chi(\Sigma) / 2} R_{\Lambda_{+}}\left(q^{1 / 2}-q^{-1 / 2}\right),
$$

for any $q$ that is a power of a prime number. Satisfying condition (1) in Strategy 1 means that the polynomial on the right side of the inequality has fewer terms than the polynomial on the left side of the inequality. If following this approach, it may be helpful to start by first finding a pair of positive integer coefficient polynomials that satisfy this inequality and condition (1) at the same time. One can start with checking the ruling polynomials of small crossing number Legendrian knots on [CN13].

\subsection{Candidates for Non-decomposable Lagrangian Concordances from}

Topology. Observe that any decomposable Lagrangian concordance will be a smooth ribbon concordance. Thus it is potentially possible to use known obstructions to ribbon concordances to find examples of smooth knots whose Legendrian representatives cannot be connected by a decomposable Lagrangian 
concordance: constructing a Lagrangian concordance between very stabilized Legendrian representatives of these knot types, via the combinatorial techniques of Section 3 or geometric techniques of Section 4, will give an example of an exact Lagrangian concordance between knots that cannot be connected by a decomposable Lagrangian concordance.

For example, it is known Gor81, Zem19, LZ19] that the only knot that admits a ribbon concordance to the unknot is the unknot itself. This has as a corollary the following obstruction to a decomposable Lagrangian concordance.

Theorem 14 ([CNS16, Theorem 3.2]). If $\Lambda_{-}$is topologically non-trivial and $\Lambda_{+}$is topologically an unknot, then there is no decomposable Lagrangian concordance from $\Lambda_{-}$to $\Lambda_{+}$.

Example 15. To illustrate this theorem, here is a possible low crossing number Legendrian knot to examine as $\Lambda_{-}$. Consider the topological knot $6_{1}$ which is slice and ribbon. Its maximum $t b$ Legendrian representative $\Lambda_{66_{1}}$ (see Figure 19) has $t b=-5$ and $r=0$. The DGA of this Legendrian $\mathcal{A}\left(\Lambda_{6_{1}}\right)$ admits an augmentation, and thus $\Lambda_{61}$ does not admit a Lagrangian cap; see obstructions (6) in Section 2.3. Since we are trying to construct a Legendrian $\Lambda_{-}$that could be Lagrangian concordant to a stabilized unknot, which might have a Lagrangian cap, we will add some stabilizations that will prevent augmentations and thereby allow the possibility of a Lagrangian cap. If we now add a positive and a negative stabilization to $\Lambda_{6_{1}}$, we get a knot $\Lambda_{6_{1}}^{ \pm}$with $t b=-7$ and $r=0$, which has no augmentation and is still topologically the knot $6_{1}$. If, by a sequence of moves in Section 3 , one can construct a concordance from $\Lambda_{6_{1}}^{ \pm}$to the $t b=-7$ stabilized unknot, then by Theorem 14 this Lagrangian concordance will not be decomposable; see Figure 20. In fact, one can stabilize $\Lambda_{6_{1}}$ as many times as we wish resulting in $t b\left(\Lambda_{-}\right)=t$ and $r\left(\Lambda_{-}\right)=r$ and try, using the combinatorial constructions of Section 3, to construct a Lagrangian concordance to $\Lambda_{+}$, where $\Lambda_{+}$is a Legendrian unknot with $t b\left(\Lambda_{+}\right)=t$ and $r\left(\Lambda_{+}\right)=r$. If possible, such a construction would prove the existence of a non-decomposable Lagrangian concordance.

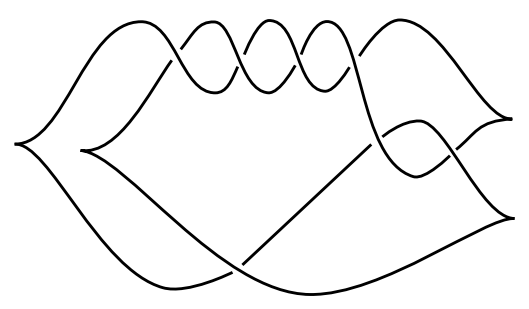

Figure 19. Front diagram of $\Lambda_{6_{1}}$.

There are additional results from topology that give obstructions to the existence of ribbon concordances. For example, as shown by Gilmer Gil84] and generalized by Friedl and Powell [FPar], if $K_{-}$is ribbon concordant to 


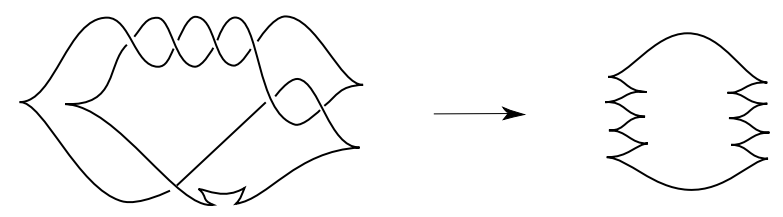

Figure 20. Any Lagrangian concordance from the doubly stabilized $\Lambda_{61}$ to the $t b=-7, r=0$ Legendrian unknot would necessarily be non-decomposable.

$K_{+}$, then the Alexander polynomial of $K_{-}$divides the Alexander polynomial of $K_{+}$. We can invoke these results in a strategy to show the existence of non-decomposable Lagrangian concordances.

Strategy 2. (1) Use results from smooth topology to find examples of smooth knots $K_{ \pm}$such that $K_{-}$is not ribbon concordant to $K_{+}$.

(2) For any pair of Legendrian representatives $\Lambda_{ \pm}$of the knot type $K_{ \pm}$, even highly stabilized, use a combination of the combinatorial moves described in Section 3 to construct a Lagrangian concordance from $\Lambda_{-}$ to $\Lambda_{+}$.

The example with the knot $6_{1}$ given above is a concrete example to try to apply this strategy with $K_{-}=6_{1}$ and $K_{+}$being an unknot. A possible example when $K_{+}$is non-trivial is the following.

Example 16. Let $K_{-}$be the connect sum of the right- and left-handed trefoils, $K_{-}=T_{r} \# T_{l}$, and let $K_{+}$be the connect sum of the figure 8 knot with itself, $K_{+}=F_{8} \# F_{8}$. These knots are concordant but there is no ribbon concordance from $K_{-}$to $K_{+}$, as first shown by Gordon [Gor81. Choose Legendrian representatives $\Lambda_{ \pm}$of $K_{ \pm}$such that $t b\left(\Lambda_{-}\right)=t b\left(\Lambda_{+}\right)$and $r\left(\Lambda_{-}\right)=r\left(\Lambda_{+}\right)$; note that $\Lambda_{ \pm}$can be very stabilized. If we can construct a Lagrangian concordance from $\Lambda_{-}$to $\Lambda_{+}$, via the combinatorial moves of Section 3 , then we will have shown the existence of a pair of Legendrians that are (exactly, orientably) Lagrangian concordant but cannot be connected by a decomposable Lagrangian concordance.

Remark 8. Some known obstructions to ribbon concordance are, in fact, obstructions to generalizations of ribbon concordance, namely strong homotopy ribbon concordance and homotopy ribbon concordance. A strong homotopy ribbon concordance is one whose complement is ribbon, i.e., can be built with only 1-handles and 2-handles. A homotopy ribbon concordance from $K_{-}$to $K_{+}$is a concordance where the induced map on $\pi_{1}$ of the complement of $K_{-}$(resp. $K_{+}$) injects (resp. surjects) into $\pi_{1}$ of the complement of the concordance. Gordon Gor81] showed that

$$
\begin{aligned}
\text { ribbon concordant } & \Longrightarrow \text { strong homotopy ribbon concordant } \\
& \Longrightarrow \text { homotopy ribbon concordant. }
\end{aligned}
$$


There have been a number of recent results obstructing (homotopy or strong homotopy) ribbon concordances from Heegaard-Floer and Khovanov homology [Zem19, LZ19, MZer, GL20]; these results play an important role in Strategy 2 .

\subsection{Candidates for Non-decomposable Lagrangian Cobordisms from}

GRID Invariants. Some candidates for non-decomposable Lagrangian cobordisms of higher genus come from knot Floer homology. Using the grid formulation of knot Floer homology OST08, Ozsváth, Szabó, and Thurston defined Legendrian invariants of a Legendrian link $\Lambda \subset \mathbb{R}^{3}$, called GRID invariants, which are elements in the hat flavor of knot Floer homology of $\Lambda \subset-S^{3}$ :

$$
\widehat{\lambda}^{+}(\Lambda), \widehat{\lambda}^{-}(\Lambda) \in \widehat{H F K}\left(-S^{3}, \Lambda\right) .
$$

For more background, see OST08, MOS09].

Baldwin, Lidman, and Wong [BLWar] have shown that these GRID invariants can be used to obstruct the existence of decomposable Lagrangian cobordisms.

Theorem 17 ([BLWar, Theorem 1.2] ). Suppose that $\Lambda_{ \pm}$are Legendrian links in $\mathbb{R}^{3}$ such that either

(1) $\hat{\lambda}^{+}\left(\Lambda_{+}\right)=0$ and $\widehat{\lambda}^{+}\left(\Lambda_{-}\right) \neq 0$, or

(2) $\widehat{\lambda}^{-}\left(\Lambda_{+}\right)=0$ and $\widehat{\lambda}^{-}\left(\Lambda_{-}\right) \neq 0$.

Then there is no decomposable Lagrangian cobordism from $\Lambda_{-}$to $\Lambda_{+}$.

Remark 9. By [BVVV13], in the standard contact manifold $\mathbb{R}^{3}$, the GRID invariants agree with the LOSS invariant [LOSS09]. The LOSS invariant is functorial on Lagrangian concordances by [BS18, BSar]. Thus Theorem 17 would also obstruct the existence of general Lagrangian concordances and not only the decomposable ones. To find non-decomposable cobordisms using obstructions from [BLWar], we should focus on non-zero genus cobordisms.

Using the facts that the GRID invariants are non-zero for the $t b=-1$ Legendrian unknot and that $\widehat{\lambda}^{+}\left(\Lambda_{+}\right)$(resp. $\widehat{\lambda}^{-}\left(\Lambda_{+}\right)$) vanish for positively (negatively) stabilized Legendrian links, Theorem 17 gives the following corollary.

Corollary 18 ([BLWar, Corollaries 1.3, 1.4]). (1) If $\Lambda \subset \mathbb{R}^{3}$ is a Legendrian link such that $\widehat{\lambda}^{+}(\Lambda)=0$ or $\widehat{\lambda}^{-}(\Lambda)=0$, then there is no decomposable Lagrangian filling of $\Lambda$.

(2) Suppose $\Lambda_{ \pm}$are Legendrian links such that either

(a) $\hat{\lambda}^{+}\left(\Lambda_{-}\right) \neq 0$ and $\Lambda_{+}$is the positive stabilization of a Legendrian link, or

(b) $\widehat{\lambda}^{-}\left(\Lambda_{-}\right) \neq 0$ and $\Lambda_{+}$is the negative stabilization of a Legendrian link.

Then there is no decomposable Lagrangian cobordism from $\Lambda_{-}$to $\Lambda_{+}$.

This provides another strategy to show the existence of Legendrians $\Lambda_{ \pm}$ that are Lagrangian cobordant but cannot be connected by a decomposable Lagrangian cobordism. 
Strategy 3. (1) Find Legendrians $\Lambda_{ \pm}$satisfying the GRID invariants conditions of Corollary 18 and Theorem 17 such that there are no known obstructions, as described in Section [2.3, to the existence of a Lagrangian cobordism from $\Lambda_{-}$to $\Lambda_{+}$.

(2) Use a combination of the combinatorial moves described in Section 3 to construct a Lagrangian cobordism from $\Lambda_{-}$to $\Lambda_{+}$.

Example 19. Concrete examples mentioned in [BLWar, Section 4.1] can be used for Strategy 3 . Let $\Lambda_{0}, \Lambda_{1}$ be the Legendrian $m\left(10_{132}\right)$ knots and Legendrian $m\left(12 n_{200}\right)$ knots shown in [NOT08, Figures 2 and 3]. Modify them with a pattern shown in [BLWar, Figure 13] to get $\Lambda_{0}^{\prime}$ and $\Lambda_{1}^{\prime}$, which are of knot type $m\left(12 n_{199}\right)$ and $m\left(14 n_{5047}\right)$ (or its mirror), respectively. For $i, j=0,1$ we have $t b\left(\Lambda_{i}^{\prime}\right)=t b\left(\Lambda_{i}\right)+2$ and $r\left(\Lambda_{i}^{\prime}\right)=r\left(\Lambda_{i}\right)$. There is no decomposable Lagrangian cobordism from

(1) $\Lambda_{0}$ to $\Lambda_{1}^{\prime}$, or

(2) $\Lambda_{1}$ to $\Lambda_{0}^{\prime}$.

If we can construct, using the combinatorial techniques of Section 3 , a Lagrangian cobordism (necessarily of genus 1 ) from $\Lambda_{0}$ to $\Lambda_{1}^{\prime}$ or from $\Lambda_{1}$ to $\Lambda_{0}^{\prime}$, then we will have found a non-decomposable Lagrangian cobordisms.

Example 20. In [BLWar, Section 4.3], the authors provide an infinite family of pairs of Legendrian knots where there does not exist a decomposable Lagrangian cobordism between them.

Remark 10. In Strategies 2 and 3, we emphasized the construction of Lagrangian cobordisms using the combinatorial techniques of Section 3. It would be interesting to know if the geometric constructions of Section 4 could also be used to show the existence of a Lagrangian concordance/cobordism from the theory of normal rulings, topology, or grid invariants, that are known to not be decomposable.

5.4. Non-decomposable Candidates through Surgery. An additional strategy to show the existence of a non-decomposable Lagrangian filling comes from understanding properties of the contact manifold that is obtained from surgery on the Legendrian knot. In particular, Conway, Etnyre, and Tosun CETar have detected a relationship between Lagrangian fillings of a Legendrian and symplectic fillings of the contact manifold obtained by performing a particular type of surgery on the Legendrian.

Theorem 21 ([CETar, Theorem 1.1]). There is a Lagrangian disk filling of $\Lambda_{+}$if and only if the contact +1 -surgery on $\Lambda_{+} \subset \mathbb{R}^{3} \subset S^{3}$ produces a contact manifold that is strongly symplectically fillable. If $\Lambda_{+}$has a decomposable Lagrangian filling, then the filling can be taken to be Stein.

In fact, CETar also shows that a filling will be a Stein filling if and only if $\Lambda_{+}$bounds a regular Lagrangian disk: a Lagrangian disk is regular if there is a Liouville vector field that is tangent to the disk. Any decomposable Lagrangian filling is regular. 
We now see another strategy to construct a non-decomposable Lagrangian filling.

Strategy 4. Find a Legendrian $\Lambda$ such that the +1 -surgery on $\Lambda$ produces a contact manifold that is strongly symplectically fillable but does not admit a Stein filling.

An issue with this approach is a lack of examples: there are very few manifolds which carry strongly fillable but not Stein fillable contact structures. The main examples are the $1 / n$ surgeries on the positive and negative trefoils; see works by Ghiggini Ghi05] and Tosun [Tos20]. However it is not obvious whether any of these contact structures are a contact +1 surgery on a Legendrian knot in $S^{3}$.

\section{Conclusion}

The desire to understand the flexibility and rigidity of Lagrangian submanifolds has led to a great deal of interesting research in symplectic topology. Similarly, trying to understand constructions of and obstructions to Lagrangian cobordisms has led to many interesting results. At this point, we have few concrete answers to the Motivating Questions stated in our Introduction. In particular, regarding Motivating Question (1), there are presently many candidates for Legendrians $\Lambda_{ \pm}$that can be connected by a Lagrangian cobordism but not by a decomposable Lagrangian cobordism: by understanding all the obstructions to Lagrangian cobordisms, one can come up with some good candidates. When trying and failing to construct a Lagrangian cobordism between a given pair, one may gain intuition for additional obstructions to Lagrangian cobordisms that are waiting to be discovered.

\section{REFERENCES}

[BLWar] John A Baldwin, Tye Lidman, and C-M Michael Wong. Lagrangian cobordisms and Legendrian invariants in knot Floer homology. Michigan Mathematical Journal, to appear.

[BS18] John A. Baldwin and Steven Sivek. Invariants of Legendrian and transverse knots in monopole knot homology. J. Symplectic Geom., 16(4):959-1000, 2018.

[BSar] John A Baldwin and Steven Sivek. On the equivalence of contact invariants in sutured Floer homology theories. Geometry \& Topology, to appear.

[BST15] F. Bourgeois, J. Sabloff, and L. Traynor. Lagrangian cobordisms via generating families: Construction and geography. Algebr. Geom. Topol., 15(4):2439-2477, 2015.

[BVVV13] John A. Baldwin, David Shea Vela-Vick, and Vera Vértesi. On the equivalence of Legendrian and transverse invariants in knot Floer homology. Geom. Topol., 17(2):925-974, 2013.

[CDRGG] Baptiste Chantraine, Georgios Dimitroglou Rizell, Paolo Ghiggini, and Roman Golovko. Floer homology and Lagrangian concordance. In Proceedings of $21^{\text {st }}$ G okova Geometry-Topology Conference, pages 76-113.

[CDRGG20] Baptiste Chantraine, Georgios Dimitroglou Rizell, Paolo Ghiggini, and Roman Golovko. Floer theory for Lagrangian cobordisms. J. Differential Geom., $114(3): 393-465,2020$. 
[CE12] Kai Cielieback and Yakov Eliashberg. From Stein to Weinstein and back: Symplectic geometry of affine complex manifolds, volume 59. AMS Colloquium Publications, 2012.

[CETar] James Conway, John B. Etnyre, and Bülent Tosun. Symplectic fillings, contact surgeries, and Lagrangian disks. International Mathematics Research Notices, to appear.

[Cha10] Baptiste Chantraine. Lagrangian concordance of Legendrian knots. Algebr. Geom. Topol., 10(1):63-85, 2010.

[Cha12] Baptiste Chantraine. Some non-collarable slices of Lagrangian surfaces. Bull. Lond. Math. Soc., 44(5):981-987, 2012.

[Cha15a] B. Chantraine. A note on exact Lagrangian cobordisms with disconnected Legendrian ends. Proc. Amer. Math. Soc., 143(3):1325-1331, 2015.

[Cha15b] Baptiste Chantraine. Lagrangian concordance is not a symmetric relation. Quantum Topol., 6(3):451-474, 2015.

[Che02] Yu. V. Chekanov. Invariants of Legendrian knots. In Proceedings of the International Congress of Mathematicians, Vol. II (Beijing, 2002), pages 385-394. Higher Ed. Press, Beijing, 2002.

[CN13] Wutichai Chongchitmate and Lenhard Ng. An atlas of Legendrian knots. Exp. Math., 22(1):26-37, 2013.

[CNS16] Christopher Cornwell, Lenhard Ng, and Steven Sivek. Obstructions to Lagrangian concordance. Algebr. Geom. Topol., 16(2):797-824, 2016.

$\left[\mathrm{CSLL}^{+} 20\right]$ Orsola Capovilla-Searle, Noémie Legout, Maÿlis Limouzineau, Emmy Murphy, Yu Pan, and Lisa Traynor. Obstructions to reversing Lagrangian surgery in Lagrangian fillings. In preparation, 2020.

[DR15] Georgios Dimitroglou Rizell. Exact Lagrangian caps and non-uniruled Lagrangian submanifolds. Ark. Mat., 53(1):37-64, 2015.

[DR16] G. Dimitroglou Rizell. Lifting pseudo-holomorphic polygons to the symplectisation of $P \times \mathbb{R}$ and applications. Quantum Topol., 7(1):29-105, 2016.

[EES05] Tobias Ekholm, John Etnyre, and Michael Sullivan. Non-isotopic Legendrian submanifolds in $\mathbb{R}^{2 n+1}$. J. Differential Geom., 71(1):85-128, 2005.

[EES09] T. Ekholm, J. B. Etnyre, and J. M. Sabloff. A duality exact sequence for Legendrian contact homology. Duke Math. J., 150(1):1-75, 2009.

[EG98] Yasha Eliashberg and Misha Gromov. Lagrangian intersection theory: finitedimensional approach. In Geometry of differential equations, volume 186 of Amer. Math. Soc. Transl. Ser. 2, pages 27-118. Amer. Math. Soc., Providence, RI, 1998.

[EGH00] Y. Eliashberg, A. Givental, and H. Hofer. Introduction to symplectic field theory. Number Special Volume, Part II, pages 560-673. 2000. GAFA 2000 (Tel Aviv, 1999).

[EHK16] Tobias Ekholm, Ko Honda, and Tamás Kálmán. Legendrian knots and exact Lagrangian cobordisms. J. Eur. Math. Soc. (JEMS), 18(11):2627-2689, 2016.

[Ekh12] T. Ekholm. Rational SFT, linearized Legendrian contact homology, and Lagrangian Floer cohomology. In Perspectives in analysis, geometry, and topology, volume 296 of Progr. Math., pages 109-145. Birkhäuser/Springer, New York, 2012.

[Eli98] Yakov Eliashberg. Invariants in contact topology. In Proceedings of the International Congress of Mathematicians, Vol. II (Berlin, 1998), number Extra Vol. II, pages 327-338, 1998.

[EP96] Yakov Eliashberg and Leonid Polterovich. Local Lagrangian 2-knots are trivial. Annals of Math, 144:1:61-76, 1996. 
[Etn05] John B. Etnyre. Legendrian and transversal knots. In Handbook of knot theory, pages 105-185. Elsevier B. V., Amsterdam, 2005.

[FI04] Dmitry Fuchs and Tigran Ishkhanov. Invariants of Legendrian knots and decompositions of front diagrams. Mosc. Math. J., 4(3):707-717, 783, 2004.

[FPar] Stefan Friedl and Mark Powell. Homotopy ribbon concordance and Alexander polynomials. Archiv der Mathematik, to appear.

[FR11] D. Fuchs and D. Rutherford. Generating families and Legendrian contact homology in the standard contact space. J. Topol., 4(1):190-226, 2011.

[Fuc03] Dmitry Fuchs. Chekanov-Eliashberg invariant of Legendrian knots: existence of augmentations. J. Geom. Phys., 47(1):43-65, 2003.

[Ghi05] Paolo Ghiggini. Strongly fillable contact 3-manifolds without Stein fillings. Geom. Topol., 9:1677-1687, 2005.

[Gil84] Patrick M. Gilmer. Ribbon concordance and a partial order on S-equivalence classes. Topology and its Applications, 18(2):313 - 324, 1984.

[GJ19] Marco Golla and András Juhász. Functoriality of the EH class and the LOSS invariant under Lagrangian concordances. Algebr. Geom. Topol., 19(7):36833699, 2019.

[GL20] Onkar Singh Gujral and Adam Simon Levine. Khovanov homology and cobordisms between split links. arXiv preprint arXiv:2009.03406, 2020.

[Gor81] C. McA. Gordon. Ribbon concordance of knots in the 3-sphere. Math. Ann., 257(2):157-170, 1981.

[Gro85] M. Gromov. Pseudoholomorphic curves in symplectic manifolds. Invent. Math., 82:307-347, 1985.

[GSY20] Roberta Guadagni, Joshua M. Sabloff, and Matthew Yacavone. Legendrian satellites and decomposable cobordisms. In preparation, 2020.

[JT06] J. Jordan and L. Traynor. Generating family invariants for Legendrian links of unknots. Algebr. Geom. Topol., 6:895-933 (electronic), 2006.

[Lin16] Francesco Lin. Exact Lagrangian caps of Legendrian knots. J. Symplectic Geom., 14(1):269-295, 2016.

[LOSS09] Paolo Lisca, Peter Ozsváth, András I. Stipsicz, and Zoltán Szabó. Heegaard Floer invariants of Legendrian knots in contact three-manifolds. J. Eur. Math. Soc. (JEMS), 11(6):1307-1363, 2009.

[LZ19] Adam Simon Levine and Ian Zemke. Khovanov homology and ribbon concordances. Bull. Lond. Math. Soc., 51(6):1099-1103, 2019.

[MOS09] Ciprian Manolescu, Peter Ozsváth, and Sucharit Sarkar. A combinatorial description of knot Floer homology. Ann. of Math. (2), 169(2):633-660, 2009.

[MZer] Maggie Miller and Ian Zemke. Knot Floer homology and strongly homotopyribbon concordances. Mathematical Research Letters, To apper.

[Ng01] Lenhard Lee Ng. Invariants of Legendrian links. ProQuest LLC, Ann Arbor, MI, 2001. Thesis (Ph.D.)-Massachusetts Institute of Technology.

[NOT08] Lenhard Ng, Peter Ozsváth, and Dylan Thurston. Transverse knots distinguished by knot Floer homology. J. Symplectic Geom., 6(4):461-490, 2008.

[NR13] Lenhard Ng and Daniel Rutherford. Satellites of Legendrian knots and representations of the Chekanov-Eliashberg algebra. Algebr. Geom. Topol., 13(5):3047-3097, 2013.

[NS06] Lenhard L. Ng and Joshua M. Sabloff. The correspondence between augmentations and rulings for Legendrian knots. Pacific J. Math., 224(1):141-150, 2006.

[NT04] L. Ng and L. Traynor. Legendrian solid-torus links. J. Symplectic Geom., 2(3):411-443, 2004. 
[Oan15] Alexandru Oancea. From Stein to Weinstein and back. Symplectic geometry of affine complex manifolds [book review of MR3012475]. Bull. Amer. Math. Soc. (N.S.), 52(3):521-530, 2015.

[OST08] Peter Ozsváth, Zoltán Szabó, and Dylan Thurston. Legendrian knots, transverse knots and combinatorial Floer homology. Geom. Topol., 12(2):941-980, 2008.

[Pan17] Yu Pan. The augmentation category map induced by exact Lagrangian cobordisms. Algebr. Geom. Topol., 17(3):1813-1870, 2017.

[PC05] P. E. Pushkar and Yu. V. Chekanov. Combinatorics of fronts of Legendrian links, and Arnold's 4-conjectures. Uspekhi Mat. Nauk, 60(1(361)):99-154, 2005 .

[Rit09] Alexander F. Ritter. Novikov symplectic homology and exact Lagrangian embeddings. Geom. Topol., 13(2):943-978, 2009.

[Rud97] L. Rudolph. The slice genus and the Thurston-Bennequin invariant of a knot. Proc. Amer. Math. Soc., 125(10):3049-3050, 1997.

[Sab05] Joshua M. Sabloff. Augmentations and rulings of Legendrian knots. Int. Math. Res. Not., (19):1157-1180, 2005.

[Sau04] Denis Sauvaget. Curiosités lagrangiennes en dimension 4. Ann. Inst. Fourier (Grenoble), 54(6):1997-2020 (2005), 2004.

[ST13] Joshua M. Sabloff and Lisa Traynor. Obstructions to Lagrangian cobordisms between Legendrians via generating families. Algebr. Geom. Topol., 13:2733 2797, 2013.

[Tos20] Bülent Tosun. Tight small Seifert fibered manifolds with $e_{0}=-2$. Algebr. Geom. Topol., 20(1):1-27, 2020.

[Tra01] Lisa Traynor. Generating function polynomials for Legendrian links. Geom. Topol., 5:719-760, 2001.

[Zem19] Ian Zemke. Knot Floer homology obstructs ribbon concordance. Ann. of Math. (2), 190(3):931-947, 2019. 